\title{
On the minimizers of the Ginzburg-Landau energy for high kappa: the axially symmetric case
}

\author{
by
}

\author{
Amandine AFTALION
}

DMI, École Normale Supérieure, 45, rue d'Ulm, 75230 Paris cedex 05

\begin{abstract}
The Ginzburg-Landau theory of superconductivity is examined in the case of a special geometry of the sample, the infinite cylinder. We restrict to axially symmetric solutions and consider models with and without vortices. First putting the Ginzburg-Landau parameter $\kappa$ formally equal to infinity, the existence of a minimizer of this reduced Ginzburg-Landau energy is proved. Then asymptotic behaviour for large $\kappa$ of minimizers of the full Ginzburg-Landau energy is analyzed and different convergence results are obtained. Our main result states that, when $\kappa$ is large, the minimum of the energy is reached when there are about $\kappa$ vortices at the center of the cylinder. Numerical computations illustrate the various behaviours.

(C) 1999 L'Association Publications de l'Institut Henri Poincaré. Published by Elsevier B.V. All rights reserved
\end{abstract}

RÉSUMÉ. - Le modèle de Ginzburg-Landau des supraconducteurs est étudié dans le cas d'une géométrie cylindrique pour des solutions radiales, avec et sans vortex. Quand le paramètre de Ginzburg-Landau $\kappa$ est infini, on prouve l'existence d'un minimum pour une énergie réduite. Puis on étudie le comportement asymptotique des minimiseurs pour $\kappa$ grand et on obtient différents résultats de convergence. On montre en particulier que l'énergie est minimum pour une configuration où le nombre de vortex au centre du cylindre est de l'ordre de $\kappa$. Des calculs numériques illustrent les divers comportements.

(C) 1999 L'Association Publications de l'Institut Henri Poincaré. Published by Elsevier B.V. All rights reserved

Classification A.M.S. 1991 : 82D55, 35Q55

Annales de l'Institut Henri Poincaré - Analyse non linéaire - 0294-1449 Vol. 16/99/06/ 


\section{INTRODUCTION}

The superconductivity of certain metals is characterized at very low temperatures by the loss of electrical resistance and the expulsion of the exterior magnetic field. Superconducting currents in the material, which exclude the magnetic field, are due to the existence of pairs of electrons of opposite sign and momentum, the Cooper pairs. In the GinzburgLandau model, the electromagnetic properties of the material are completely described by the magnetic potential vector $\mathbf{A}(\mathbf{H}=$ curl $\mathbf{A}$ being the magnetic field) and the complex-valued order parameter $\psi$ (see [11]. [13] or [16] for instance). In fact, $\psi$ is an averaged wave function of the superconducting electrons; its phase is related to the current in the superconductor and its modulus to the density of superconducting carriers: $|\psi|=0$ when the sample is wholly normal and $|\psi|=1$ when it is wholly superconducting. The basic thermodynamic postulate of the GinzburgLandau theory says that a stable superconducting sample is in a state such that its Gibbs free energy is a minimum. The nondimensionalized form of this energy is given by:

$$
E_{\kappa}(\psi, \mathbf{A})=\int_{\Omega}\left|\left(\frac{1}{\kappa} \nabla-i \mathbf{A}\right) \psi\right|^{2}+\frac{1}{2}\left(|\psi|^{2}-1\right)^{2}+\left(\operatorname{curl} \mathbf{A}-\mathbf{H}_{0}\right)^{2} d \Omega
$$

where $\Omega$ is a domain in $\mathbb{R}^{n}(n=1,2$ or 3$)$ representing the region occupied by the superconducting sample, $\mathbf{H}_{0}$ is the given applied magnetic field, and $\kappa$ is a material parameter called the Ginzburg-Landau parameter. This parameter is the ratio of $\lambda$, the penetration depth of the magnetic field to $\xi$, the coherence length, which is the characteristic length of variation of $\psi$. The value of $\kappa$ determines the type of superconductor: $\kappa \leq 1 / \sqrt{2}$ describes what is known as a type I superconductor and $\kappa \geq 1 / \sqrt{2}$ as a type II. Type I superconductors are either normally conducting (normal state) or superconducting according to the value of the magnetic field, while for type II, a third state appears, called the mixed state: in the mixed state, the superconducting and the normal states coexist in what is usually called filaments or vortices. At the center of the vortex, the order parameter vanishes, so the material is normal; the vortex is circled by a superconducting current carrying with it a quantized amount of magnetic flux. Macroscopic models of superconducting vortices have been formulated in [9], [10] and the existence and behaviour of vortex solutions to the Ginzburg-Landau equations have been widely studied. See [2], [4], [5], [7], [8], [12], [14] for instance.

In this paper, we study the minimization of the Ginzburg-Landau energy when the parameter $\kappa$ is large, for a special geometry of the sample, 
the infinite cylinder. Thus, we restrict to axially symmetric solutions. The previous paper [1] deals with the one-dimensional case. Our motivation was the study made in [3] of the solutions of the Ginzburg-Landau system with infinite $\kappa$.

This paper is organized as follows. First of all, we study the asymptotic behaviour of minimizers under the constraint that vortices do not exist: we put formally $\kappa$ equal to infinity in the energy and study the minimizers of this reduced form; this will enable us to show convergence of GinzburgLandau energy minimizers as $\kappa$ tends to infinity. We especially prove a uniqueness property of the solutions of the system for infinite $\kappa$, which extends a result of [3]. The section ends with numerical results. The same type of work is made in the next section for the model with vortices. Our main result states that when $\kappa$ is large the minimum of the energy is reached when there are about $\kappa$ vortices at the center of the cylinder.

Let us recall from [13] the main properties of the two dimensional Ginzburg-Landau model. We assume that the superconducting sample is an infinite cylinder. When the magnetic field is parallel to the axis of the cylinder, $\mathbf{H}_{0}=\left(0,0, H_{0}\right)$, one can assume that both $\psi$ and $\mathbf{A}$ are uniform in the z-direction. The state of the superconductor is described by the pair $(\psi, \mathbf{A})$ that minimizes $E_{\kappa}$, where $\Omega$ is a regular bounded domain in $\mathbb{R}^{2}$. Let us define

$$
H_{n}^{1}(\operatorname{div})=\left\{\mathbf{A} \in H^{1}\left(\Omega, \mathbb{R}^{3}\right), \operatorname{div} \mathbf{A}=0 \text { and } \mathbf{A} \cdot \mathbf{n}=0\right\} .
$$

We can choose $\|$ curl $\mathbf{A} \|_{L^{2}}$ as a norm on $H_{n}^{1}(d i v)$.

Definition 1.1. - $(\psi, \mathbf{A})$ and $(\phi, \mathbf{B})$ are said to be gauge-equivalent if there exists $\theta \in H^{2}(\Omega, \mathbb{R})$ such that $\psi=\phi e^{i \theta}$ and $\mathbf{B}=\mathbf{A}-(1 / \kappa) \nabla \theta$. Then, the energy $E_{\kappa}$ is preserved by this transformation.

THEOREM 1.2. - A minimizer of $E_{\kappa}$ over $H^{1}(\Omega, \mathbb{R}) \times H^{1}\left(\Omega, \mathbb{R}^{3}\right)$ is gauge equivalent to a minimizer of $E_{\kappa}$ over $H^{1}(\Omega, \mathbb{R}) \times H_{n}^{1}($ div $)$.

THEOREM 1.3. - There exists a minimum $(\psi, \mathbf{A})$ of $E_{\kappa}$ over $H^{1} \times H_{n}^{1}(d i v)$. It is a solution of:

$$
\begin{aligned}
& \left(\frac{1}{\kappa} \nabla-i \mathbf{A}\right)^{2} \psi=\psi\left(1-\left.|\psi|\right|^{2}\right) \text { in } \Omega \\
& \Delta \mathbf{A}=\frac{i}{2 \kappa}\left(\psi^{*} \nabla \psi-\psi \nabla \psi^{*}\right)+\mathbf{A}|\psi|^{2} \text { in } \Omega \\
& \frac{\partial \psi}{\partial n}=0 \quad \text { on } \partial \Omega \\
& \text { curl } \mathbf{A}=\mathbf{H}_{0} \quad \text { on } \partial \Omega .
\end{aligned}
$$

Vol. 16, n 6-1999. 
Proposition 1.4. - If $(\psi, \mathbf{A})$ is a minimizer of $E_{\kappa}$ then $|\psi| \leq 1$ a.e.

If $\Omega$ is a general domain, the gauge-invariance property does not remain in $E_{\infty}$, (the energy obtained when $\kappa$ is formally put equal to infinity), so that we do not have a global minimum of the energy. Hence we shall now restrict ourselves to a ball.

\section{THE CASE WITHOUT VORTICES}

When $\Omega$ is assumed to be a ball, we use polar coordinates and restrict to radial solutions. In this section, we make the extra assumption that no vortices exist (so $\psi$ is never equal to zero) and we look for:

$$
\psi(r, \theta)=f(r) \quad f(r) \in \mathbb{R}_{+}^{*}, \quad \text { and } \quad \mathbf{A}(r, \theta)=Q(r) \mathbf{e}_{\theta} .
$$

We automatically have $\operatorname{div} \mathbf{A}=0$, which is the major interest of this gauge. So $\|$ curl $\mathbf{A}\left\|_{L^{2}\left(B_{R}\right)}=\right\|(1 / r)(r Q)^{\prime} \|_{L^{2}\left(B_{R}\right)}$ is a norm on $H^{1}\left(B_{R}\right)$. Since we want $(\psi, \mathbf{A})$ in $H^{1} \times H^{1}$, we define:

$$
\begin{aligned}
& D_{f}=\left\{f \text { radial, } f \geq 0 \text { a.e. }, f \in H^{1}\left(B_{R}, \mathbb{R}\right)\right\}, \\
& D_{Q}=\left\{\mathbf{Q} \in H^{1}\left(B_{R}, \mathbb{R}^{3}\right), \quad \exists Q(r) \text { such that } \mathbf{Q}=Q(r) \mathbf{e}_{\theta}\right\} .
\end{aligned}
$$

From now on, when we write that $Q$ is in $D_{Q}$, it will mean that $\mathbf{Q}=Q(r) \mathbf{e}_{\theta}$ is in $D_{Q}$. Notice that $\|f\|_{H^{1}\left(B_{R}\right)}$ is a norm on $D_{f}$ and $\left\|(1 / r)(r Q)^{\prime}\right\|_{L^{2}\left(B_{R}\right)}$ is a norm on $D_{Q}$. The energy can be rewritten as follows:

$$
E_{\kappa}(f, Q)=\int_{0}^{R}\left(\frac{1}{\kappa^{2}} f^{\prime 2}+\frac{1}{2}\left(f^{2}-1\right)^{2}+f^{2} Q^{2}+\left(\frac{1}{r}(r Q)^{\prime}-H_{0}\right)^{2}\right) r d r .
$$

The same method as in [1] allows us to say that:

- there exists a minimizer of $E_{\kappa}$ over $D_{f} \times D_{Q}$,

- it is a solution of

$$
\begin{aligned}
& \frac{1}{r \kappa^{2}}\left(r f^{\prime}\right)^{\prime}=f\left(f^{2}+Q^{2}-1\right) \text { in }(0, R), \\
& \left(\frac{1}{r}(r Q)^{\prime}\right)^{\prime}=f^{2} Q \quad \text { in }(0, R) \\
& f^{\prime}(R)=0 \\
& \frac{1}{R}(r Q)^{\prime}(R)=H_{0} .
\end{aligned}
$$


Equations (7)-(8) can be rewritten using the laplacian in polar coordinates:

$$
\begin{aligned}
& \frac{1}{\kappa^{2}} \Delta f=f\left(f^{2}+Q^{2}-1\right) \text { in } B_{R} \backslash\{0\} \\
& \Delta \mathbf{Q}=f^{2} \mathbf{Q} \text { in } B_{R} \backslash\{0\} .
\end{aligned}
$$

\subsection{Properties of solutions}

First of all, we notice, as in [4], that if $\mathbf{Q}$ is in $D_{Q}$, it implies that $\sqrt{r} Q \in H^{1}(0, R), Q$ is continuous on $[0, R]$ and $Q(0)=0$. More precisely, $D_{Q} \subset L^{\infty}\left(B_{R}\right)$ with

$$
\|Q\|_{L^{\infty}} \leq \frac{1}{\sqrt{2 \pi}}\left\|\frac{1}{r}(r Q)^{\prime}\right\|_{L^{2}\left(B_{R}\right)} .
$$

THEOREM 2.1. - If $(f, Q)$ is a minimizer of $E_{\kappa}$, then $f$ and $Q$ are in $C^{\infty}[0, R]$

Proof. - The previous remark and Proposition 1.4 give that $(f, Q)$ is in $L^{\infty}\left(B_{R}\right)$. Then the result follows from classical elliptic estimates on the formulation given by equations (11)-(12).

Remark. - As $f$ is regular in 0 , equation (7) implies that $f^{\prime}(0)=0$.

PROPOSITION 2.2. - If $(f, Q)$ is a minimizer of $E_{\kappa}$, then either $f \equiv 0$ or $f$ is never equal to zero.

From now on, we shall assume that $f>0$ on $(0, R]$ for a minimizer of $E_{\kappa}$. The solution $f_{0} \equiv 0$ and $Q_{0}(r)=H_{0} r / 2$ is called the normal state.

THEOREM 2.3. - If $(f, Q)$ is a minimizer of $E_{\kappa}$, then $Q$ is nondecreasing.

Proof. - Let us first show that $Q \geq 0$ on $[0, R]$. If $Q$ reached a negative minimum at $r=r_{0}$ on $(0, R)$, then we would have $Q<0$ and (8) would mean

$$
\Delta Q=Q\left(f^{2}+\frac{1}{r^{2}}\right) \leq 0
$$

on a small intervall around $r_{0}$; this is in contradiction with the Maximum Principle. Similarly, if $Q$ reached a negative minimum at $r=R$, the Hopf Lemma would imply that $Q^{\prime}(R)<0$ but then condition (10) would not be satisfied.

Now if $Q$ was not nondecreasing, we would have the existence of $r_{1}$ and $r_{2}$ in $(0, R)$ such that $r_{1}<r_{2}$ and $Q\left(r_{1}\right)>Q\left(r_{2}\right)$. It would imply Vol. $16, \mathrm{n}^{\circ}$ 6-1999. 
$Q$ reached a positive maximum on $\left(r_{1}, r_{2}\right)$. This contradicts the Maximum Principle since $\Delta Q \geq 0$ on $(0, R)$.

\subsection{Infinite Ginzburg-Landau Parameter}

We put $\kappa$ equal to infinity in the energy. We define

$$
E_{\infty}(f, Q)=\int_{0}^{R}\left(\frac{1}{2}\left(f^{2}-1\right)^{2}+f^{2} Q^{2}+\left(\frac{1}{r}(r Q)^{\prime}-H_{0}\right)^{2}\right) r d r
$$

where $f \in L_{\text {rad }}^{4}\left(B_{R}, \mathbb{R}\right)=\left\{f\right.$ radial, $\left.f \geq 0, f \in L^{4}\left(B_{R}, \mathbb{R}\right)\right\}$ and $Q \in D_{Q}$.

THEOREM 2.4. - The minimum of $E_{\infty}$ over $L_{r a d}^{4}\left(B_{R}\right) \times D_{Q}$ exists and is attained. Moreover it satisfies:

$$
\begin{aligned}
& Q^{\prime \prime}(r)+\frac{1}{r} Q^{\prime}(r)-\frac{1}{r^{2}} Q(r)=Q(r)\left(1-Q^{2}(r)\right) \mathbf{1}_{|Q| \leq 1} \text { in } B_{R} \\
& Q^{\prime}(r)+\frac{1}{r} Q(r)=H_{0} \text { for } r=R \quad \text { and } \quad|Q(r)| \geq 1 \\
& f^{2}(r)=\left(1-Q^{2}(r)\right) \mathbf{1}_{|Q| \leq 1} \text { in } B_{R}
\end{aligned}
$$

where $\mathbf{1}_{|Q| \leq 1}(x)=1$ if $|Q(x)| \leq 1$, and 0 otherwise.

The proof is almost the same as in [1]. It comes from the fact that when $Q$ is fixed, the minimum of $(1 / 2)\left(1-f^{2}\right)^{2}+f^{2} Q^{2}$ is attained for $f=0$ if $|Q| \geq 1$, and $f^{2}=1-Q^{2}$ if $|Q| \leq 1$.

THEOREM 2.5. - There exists $H_{0}^{c}$ such that

(i) for $H_{0} \leq H_{0}^{c}$, there exists a unique solution $Q$ of (14)-(15) in $D_{Q}$ and it remains smaller than 1 in $(0, R)$. If we call $\alpha\left(R, H_{0}\right)=Q(R)$, then $\alpha\left(R, H_{0}\right)$ is a continuous and increasing function of $R$ and $H_{0}$. Moreover, $\alpha\left(R, H_{0}\right)$ reaches 1 when $H_{0}=H_{0}^{c}$,

(ii) for $H_{0}>H_{0}^{c}$, there is no solution that remains smaller than 1 .

Proof. - We proceed as in [3], using a shooting method from the boundary $r=R$. For a given $\alpha \in(0,1)$, there is a unique solution $Q_{\alpha}$ of

$$
\left\{\begin{array}{l}
Q_{\alpha}^{\prime \prime}+\frac{1}{r} Q_{\alpha}^{\prime}-\frac{1}{r^{2}} Q_{\alpha}=Q_{\alpha}\left(1-Q_{\alpha}^{2}\right) \quad \text { on }(0, R), \\
Q_{\alpha}(R)=\alpha \quad \text { and } \quad Q_{\alpha}^{\prime}(R)=H_{0}-\alpha / R .
\end{array}\right.
$$

We check that (15) is satisfied when $r=R$. The three possible behaviours of $Q_{\alpha}$ are described by the following sets: 


$$
\begin{aligned}
I\left(R, H_{0}\right)= & \left\{\alpha \in(0,1), Q_{\alpha}(r) \in(0,1) \text { on }(0, R)\right\}, \\
I_{0}\left(R, H_{0}\right)= & \{\alpha \in(0,1), \\
& \left.\exists \zeta \in(0, R) \text { st } Q_{\alpha}(r) \in(0,1) \text { on }(\zeta, R) \text { and } Q_{\alpha}(\zeta)=0\right\}, \\
I_{1}\left(R, H_{0}\right)= & \{\alpha \in(0,1), \\
& \left.\exists \zeta \in(0, R) \text { st } Q_{\alpha}(r) \in(0,1) \text { on }(\zeta, R) \text { and } Q_{\alpha}(\zeta)=1\right\} .
\end{aligned}
$$

We recall from [3] that

- $I\left(R, H_{0}\right)$ corresponds to regular solutions, that is $Q_{\alpha}(0)=0$,

- $I_{0}\left(R, H_{0}\right)$ and $I_{1}\left(R, H_{0}\right)$ are open sets,

- $I_{1}\left(R, H_{0}\right) \neq \emptyset$ when $R H_{0}<1$, so it implies that $I\left(R, H_{0}\right) \neq \emptyset$ too,

- $\left(0, \frac{H_{0}}{R+2 / R}\right) \subset I_{0}\left(R, H_{0}\right)$, so for $H_{0}>R+2 / R, I\left(R, H_{0}\right)=\emptyset$.

We may also notice that $Q_{\alpha}$ cannot reach a positive local maximum while it remains between 0 and 1 , since (17) can be rewritten

$$
\Delta Q_{\alpha}-Q_{\alpha}\left(1+\frac{1}{r^{2}}-Q_{\alpha}^{2}\right)=0 .
$$

So $Q_{\alpha}$ is increasing on $\left(r_{0, \alpha}, R\right)$ where $r_{0, \alpha}$ is such that $Q_{\alpha}\left(r_{0, \alpha}\right)=0$.

It is proved in [3] that there exists $H_{0}^{*}$ such that for $H_{0}<H_{0}^{*},(14)-(15)$ has a solution that remains smaller than $1 ; H_{0}^{*}$ is obtained as the maximum of $H_{0}$ over the points $\left(R, H_{0}\right)$ in the connected component of $I_{1}$ which contains the set $\left\{\left(R, H_{0}\right)\right.$, st $\left.R H_{0}<1\right\}$. The uniqueness result there is only obtained for $R \leq 1 / \sqrt{2}$. We are going to prove that in fact $I_{0}\left(R, H_{0}\right)$ and $I_{1}\left(R, H_{0}\right)$ are connected and that $I\left(R, H_{0}\right)$ has at most one point, using an idea inspired by [17].

Let $u$ and $v$ be two different regular solutions of

$$
Q^{\prime \prime}+\frac{1}{r} Q^{\prime}-Q\left(1+\frac{1}{r^{2}}-Q^{2}\right)=0 \text { on }(0, R) \text {. }
$$

We already know that $u$ and $v$ are increasing so they can be inverted. We denote the inverse functions by $r(x)$ and $s(x)$. Using as new variables

$$
\begin{aligned}
& x=u(r) \quad \text { and } \quad U(x)=\frac{1}{2} r^{2}(x) u^{\prime 2}(r(x)), \\
& y=v(s) \text { and } \quad V(y)=\frac{1}{2} s^{2}(y) v^{\prime 2}(s(y)),
\end{aligned}
$$

we can rewrite (18) as

$$
\begin{array}{ll}
U^{\prime}(x)=x\left(1+r^{2}(x)\left(1-x^{2}\right)\right) & x \in(0, u(R)), \\
V^{\prime}(y)=y\left(1+s^{2}(y)\left(1-y^{2}\right)\right) & y \in(0, v(R)) .
\end{array}
$$

Vol. 16, $\mathrm{n}^{\circ}$ 6-1999. 
We assume $u<v \leq 1$ on $(0, \sigma)$, so that $s(x)<r(x)$ for $x \in(0, u(\sigma))$ and $u^{\prime}(0) \leq v^{\prime}(0)$. We obtain $(U-V)^{\prime}(x)>0$ and since $U(0)=V(0)=0$, we have $U(x)>V(x)$, which can be rewritten $r(x) u^{\prime}(r(x))>s(x) v^{\prime}(s(x))$ for $x \in(0, u(\sigma))$. As a consequence, for all $y \in(0, u(\sigma))$,

$$
\begin{gathered}
\int_{0}^{y} \frac{x^{2}\left(1-x^{2}\right)}{r(x) u^{\prime}(r(x))} d x<\int_{0}^{y} \frac{x^{2}\left(1-x^{2}\right)}{s(x) v^{\prime}(s(x))} d x \\
\text { or } \int_{0}^{r(y)} \frac{u^{2}(r)\left(1-u^{2}(r)\right)}{r} d r<\int_{0}^{s(y)} \frac{v^{2}(s)\left(1-v^{2}(s)\right)}{s} d s .
\end{gathered}
$$

For any solution $Q$ of (18), we now introduce

$$
F_{Q}(r)=\frac{1}{2}\left(\frac{1}{r}(r Q)^{\prime}\right)^{2}+\frac{1}{4}\left(1-Q^{2}\right)^{2}
$$

A straightforward computation, using (18), gives $F_{Q}^{\prime}(r)=(1 / r) Q^{2}\left(1-Q^{2}\right)$. Thanks to an integration from 0 to $\sigma$ of $F_{Q}^{\prime}(r)$, thanks to (20) and because $u^{\prime}(0) \leq v^{\prime}(0)$, we get

$$
\left(u^{\prime}(\sigma)+\frac{u(\sigma)}{\sigma}\right)^{2}-\left(v^{\prime}(\sigma)+\frac{v(\sigma)}{\sigma}\right)^{2}+\frac{1}{2}\left(1-u(\sigma)^{2}\right)^{2}-\frac{1}{2}\left(1-v(\sigma)^{2}\right)^{2}<0 .
$$

Therefore, two regular solutions cannot intersect before reaching 1 , otherwise we would have $u<v$ on $(0, \sigma)$ and $u(\sigma)=v(\sigma)$ with $u^{\prime}(\sigma)>v^{\prime}(\sigma)$, which contradicts (21).

We are now able to show uniqueness of regular solutions. Let $R$ be fixed. Let us assume $Q_{1}$ (resp. $Q_{2}$ ) is a solution of (17) with $\alpha=\alpha_{1}$ (resp. $\alpha_{2}$ ) and $H_{0}=H_{1}$ (resp. $H_{2}$ ). If $\alpha_{1}<\alpha_{2}$, since two regular solutions do not intersect before reaching 1 , we have $Q_{1}<Q_{2}$ on $(0, R)$. We infer from (21) that $H_{1}<H_{2}$. So there is a unique $\alpha\left(R, H_{0}\right)$ in $I\left(R, H_{0}\right)$ for each $R$ and $H_{0}$ and $\alpha\left(R, H_{0}\right)$ is an increasing function of $H_{0}$. An immediate consequence of the uniqueness is that $I_{0}\left(R, H_{0}\right)$ and $I_{1}\left(R, H_{0}\right)$ are connected sets.

Moreover, $\alpha\left(R, H_{0}\right)$ is an increasing function of $R$. Indeed, let $\alpha_{1}\left(R_{1}, H_{0}\right)<\alpha_{2}\left(R_{2}, H_{0}\right)$ be the initial data for the two regular solutions $Q_{1}$ and $Q_{2}$. We know that $Q_{1}$ and $Q_{2}$ do not intersect in $\left(0, \min \left(R_{1}, R_{2}\right)\right)$. Let us assume $Q_{1}<Q_{2}$ on $\left(0, \min \left(R_{1}, R_{2}\right)\right)$; we call $r_{2}$ the point where $Q_{2}(r)=\alpha_{1}$. We now use (20) with $y=\alpha_{1}$ which provides the following comparison result $F_{Q_{1}}\left(R_{1}\right)-F_{Q_{1}}(0)<F_{Q_{2}}\left(r_{2}\right)-F_{Q_{2}}(0)$. This yields $H_{0}<Q_{2}^{\prime}\left(r_{2}\right)+\left(1 / r_{2}\right) Q_{2}\left(r_{2}\right)$. But it is impossible since 
$Q_{2}^{\prime}(r)+(1 / r) Q_{2}(r)$ is increasing and reaches $H_{0}$ when $r=R_{2}$. So $Q_{2}<Q_{1}$ on $\left(0, \min \left(R_{1}, R_{2}\right)\right)$ and it implies $R_{1}<R_{2}$.

The continuity of $\alpha\left(R, H_{0}\right)$ follows from the fact that $I_{0}\left(R, H_{0}\right)$ and $I_{1}\left(R, H_{0}\right)$ are open and the continuous dependence of $Q$ with respect to $R$ and $H_{0}$ on any interval that does not contain zero. Indeed, if $\alpha_{0}<\alpha\left(R, H_{0}\right)<\alpha_{1}$, we have $Q_{\alpha_{0}}\left(r_{0}\right)<0$ and $Q_{\alpha_{1}}\left(r_{1}\right)>1$ for some $r_{0}$ and $r_{1}$ smaller than $R$. But these inequalities remain true for all $\left(\hat{R}, \hat{H}_{0}\right)$ in a neighbourhood of $\left(R, H_{0}\right)$, which shows $\alpha_{0}<\alpha\left(\hat{R}, \hat{H}_{0}\right)<\alpha_{1}$.

We are now able to conclude the proof. Let $R$ be fixed. For $H_{0}$ small, we know that $I_{1}\left(R, H_{0}\right)$ is nonempty, so $I\left(R, H_{0}\right)$ is nonempty too. We call

$$
H_{0}^{c}=\max \left\{H_{0} \text { such that for } H<H_{0}, I_{1}(R, H) \neq \emptyset\right\} .
$$

It can easily be seen from the continuous dependence of $I_{1}\left(R, H_{0}\right)$ with respect to $H_{0}$ that when $H_{0}$ reaches $H_{0}^{c}, \alpha\left(R, H_{0}\right)$ reaches 1 . As $\alpha\left(R, H_{0}\right)$ is an increasing function of $H_{0}$, it implies $I\left(R, H_{0}\right)$ is empty for $H_{0}>H_{0}^{c}$.

Proposition 2.6. - We have $H_{0}^{c}(R)$ is a decreasing function of $R$. Moreover

$$
\lim _{R \rightarrow \infty} H_{0}^{c}(R)=\frac{1}{\sqrt{2}} .
$$

Remark. - This is the same limit as in the one-dimensional case.

Proof. - We have shown in the proof of Theorem 2.5 that for $R_{1}<R_{2}$, $\alpha\left(R_{1}, H_{0}\right)<\alpha\left(R_{2}, H_{0}\right)$. Let $H_{0}=H_{0}^{c}\left(R_{2}\right)$, so that $\alpha\left(R_{2}, H_{0}^{c}\left(R_{2}\right)\right)=1$. It means $H_{0}^{c}\left(R_{1}\right)>H_{0}^{c}\left(R_{2}\right)$, which is the desired monotonicity property.

Let $Q$ be the regular solution of (17) with $H_{0}=H_{0}^{c}(R)$, so that $\alpha=1$. As $F_{Q}$ is increasing, we obtain $F_{Q}(0) \leq F_{Q}(R)$, that is $H_{0}^{c}(R)>1 / \sqrt{2}$. In order to get the estimate on the other side, we introduce a new energy:

$$
G_{1}(r)=\frac{1}{2} Q^{\prime 2}(r)+\frac{1}{4}\left(1-Q^{2}(r)\right)^{2}-\frac{1}{2 r^{2}} Q^{2}(r) .
$$

A simple computation, using (17) gives $G_{2}(r)=r^{3} G_{1}^{\prime}(r)=-r^{2} Q^{\prime 2}(r)+$ $Q^{2}(r)$. We now differentiate $G_{2}$ and get $G_{2}^{\prime}(r)=-2 r^{2} Q(r) Q^{\prime}(r)(1-$ $\left.Q^{2}(r)\right)$. So $G_{2}$ is decreasing and since $G_{2}(0)=0$, it means $G_{1}(0)>G_{1}(R)$ which can be rewritten

$$
H_{0}^{c}(R)<\frac{1}{R}+\sqrt{\frac{1}{2}+\frac{1}{R^{2}}} .
$$


THEOREM 2.7. - There exists a unique minimizer $\left(f_{\infty}, Q_{\infty}\right)$ of $E_{\infty}$.

For $H_{0} \leq H_{0}^{c}, Q_{\infty}$ remains smaller than 1 and $f_{\infty}^{2}=1-Q_{\infty}^{2}$.

For $H_{0}>H_{0}^{c}$, there exists a unique $R_{\infty}<R$ such that

in $\left(0, R_{\infty}\right), Q_{\infty}$ remains smaller than 1 and $f_{\infty}^{2}=1-Q_{\infty}^{2}$

while in $\left(R_{\infty}, R\right), Q_{\infty}^{\prime}+(1 / r) Q_{\infty}=H_{0}$ and $f_{\infty} \equiv 0$.

Proof. - Theorem 2.5 gives that for $H_{0} \leq H_{0}^{c}$, there is a unique $\left(f_{\infty}, Q_{\infty}\right)$ solution of (14)-(15)-(16) and $Q_{\infty}$ remains smaller than 1. For $H_{0}>H_{0}^{c}$, as we want a solution in $D_{Q}$, it means $Q_{\infty}(0)=0$ so $Q_{\infty}$ is a solution of (18) on $\left(0, R_{\infty}\right)$, with $Q_{\infty}\left(R_{\infty}\right)=1$. The radius $R_{\infty}$ is unique because $\alpha\left(R, H_{0}\right)$ is an increasing function of $R$. So there is a unique solution of (14)-(15)-(16) with $f_{\infty} \not \equiv 0$. What only remains to show is that this solution is the minimizer of $E_{\infty}$, or more precisely that the normal state (any solution defined hy $f_{0} \equiv 0$ and $Q_{0}^{\prime}+Q_{0} / r=H_{0}$ ) has a higher energy. We introduce a new energy

$$
E(r)=\frac{1}{2}\left(r Q_{\infty}^{\prime}\right)^{2}-\frac{1}{2} Q_{\infty}^{2}+\frac{1}{4} r^{2}\left(1-Q_{\infty}^{2}\right)^{2} \text { on }\left(0, R_{\infty}\right),
$$

and we call $R_{\infty}=R$ in the case $H_{0} \leq H_{0}^{c}$ with $\alpha=Q_{\infty}\left(R_{\infty}\right)$. Computing $E^{\prime}(r)$, using equation (14), gives

$$
\frac{1}{2} \int_{0}^{R_{\infty}} r\left(1-Q_{\infty}^{2}\right)^{2} d r=\frac{1}{4} R_{\infty}^{2}\left(1-\alpha^{2}\right)^{2}+\frac{1}{2} H_{0}^{2} R_{\infty}^{2}-H_{0} R_{\infty} \alpha .
$$

This and an integration by parts on $\int_{0}^{R}(1 / r)\left(\left(r Q_{\infty}\right)^{\prime}\right)^{2}$ enablc us to estimate

$$
E_{\infty}\left(f_{\infty}, Q_{\infty}\right)=\frac{1}{4} R^{2}-\frac{1}{4} R_{\infty}^{2}\left(1-\alpha^{2}\right)^{2}-\int_{0}^{R_{\infty}} r Q_{\infty}^{2}\left(1-Q_{\infty}^{2}\right) .
$$

So $E_{\infty}\left(f_{\infty}, Q_{\infty}\right)<R^{2} / 4=E_{\infty}\left(f_{0}, Q_{0}\right)$.

\subsection{Convergence of minimizers}

Proposition 2.8. - For all $H_{0}$, there exists $\kappa_{0}$ such that for $\kappa \geq \kappa_{0}$, the normal state is not a minimizer of $E_{\kappa}$.

The proof relies on energy comparisons as in [1].

THEOREM 2.9. - The whole sequence $\left(f_{\kappa}, Q_{\kappa}\right)$ of minimizers of $E_{\kappa}$ converges to the unique minimizer $\left(f_{\infty}, Q_{\infty}\right)$ of $E_{\infty}$. More precisely, when $\kappa \rightarrow \infty$,

$$
\begin{aligned}
f_{\kappa} & \rightarrow f_{\infty} \quad \text { in } L^{p}\left(B_{R}\right) \quad \forall p \quad 1 \leq p<\infty \text { and weakly in } H^{1}\left(B_{R}\right), \\
Q_{\kappa} \rightarrow Q_{\infty} & \text { in } C^{1, \alpha}\left(B_{R}\right) \quad \forall \alpha<1
\end{aligned}
$$


Proof. - It is almost the same as in [1]. We only mention the main ideas.

For $H_{0}<H_{0}^{c}$, as $\left|Q_{\infty}\right|<1, f_{\infty}$ is in $H^{1}\left(B_{R}\right)$ and we can test it in $E_{\kappa}$. We infer that $f_{\kappa}$ is bounded in $H^{1}\left(B_{R}\right)$ and $E_{\kappa}\left(f_{\kappa}, Q_{\kappa}\right)$ tends to $E_{\infty}\left(f_{\infty}, Q_{\infty}\right)$. As a consequence, $Q_{\kappa}$ is bounded in $H^{1}\left(B_{R}\right)$. So for a subsequence,

$$
\begin{array}{cccc}
f_{\kappa} \rightarrow f \quad \text { in } \quad L^{p}\left(B_{R}\right) & \forall p & 1 \leq p<\infty \text { and weakly in } H^{1}\left(B_{R}\right) \\
Q_{\kappa} \rightarrow Q \quad \text { in } \quad L^{p}\left(B_{R}\right) & \forall p & 1 \leq p<\infty \text { and weakly in } H^{1}\left(B_{R}\right)
\end{array}
$$

Using equation (12), we can improve the convergence on $Q_{\kappa}$ : classical elliptic estimates and Sobolev embeddings give that $\mathbf{Q}_{\kappa}$ is bounded in $W^{2, p}\left(B_{R}\right)$ for all finite $p$, and $Q_{\kappa}$ converges in $C^{1, \alpha}\left(B_{R}\right)$ for $\alpha<1$. We see that $E_{\infty}(f, Q)=E_{\infty}\left(f_{\infty}, Q_{\infty}\right)$ and Theorem 2.7 gives the conclusion.

For $H_{0} \geq H_{0}^{c}$, we have seen that $f_{\infty}$ is not in $H^{1}\left(B_{R}\right)$, but as in the one-dimensional case, we can find $g_{\kappa}$ in $H^{1}\left(B_{R}\right)$ such that $\lim _{\kappa \rightarrow \infty} E_{\kappa}\left(g_{\kappa}, Q_{\infty}\right)=E_{\infty}\left(f_{\infty}, Q_{\infty}\right)$. Energy comparisons give:

$$
\lim _{\kappa \rightarrow \infty} \int_{B_{R}} \frac{1}{\kappa^{2}} f_{\kappa}^{\prime 2}=0 \text { and } \lim _{\kappa \rightarrow \infty} E_{\infty}\left(f_{\kappa}, Q_{\kappa}\right)=E_{\infty}\left(f_{\infty}, Q_{\infty}\right) \text {. }
$$

As in the one-dimensional case, up to the extraction of a subsequence,

$$
\begin{aligned}
& f_{\kappa}^{2} \rightarrow f^{2} \quad \text { weakly in } L^{2}\left(B_{R}\right), \\
& Q_{\kappa} \rightarrow Q \quad \text { in } \quad C^{1, \alpha}\left(B_{R}\right),
\end{aligned}
$$

for $(f, Q) \in L^{4} \times D_{Q}$. Lower semi-continuity yields: $\liminf _{\kappa \rightarrow \infty} E_{\kappa}\left(f_{\kappa}, Q_{\kappa}\right)$ $\geq E_{\infty}(f, Q)$, so that $(f, Q)$ is the minimizer of $E_{\infty}$. Theorem 2.7 allows us to know the properties of $f$ : there exists $R_{\infty}$ with $f \equiv 0$ on $\left(0, R_{\infty}\right)$ and $f^{2}=1-Q^{2}$ on $\left(R_{\infty}, R\right)$. Then the result of convergence follows as in the one-dimensional case.

\subsection{Numerical Study}

We want to compute solutions of the Ginzburg-Landau system such that $f$ is positive. Instead of solving the system (7)-(8)-(9)-(10), we define $S(r)=r Q(r)$, choose $\epsilon$ small and solve:

$$
\begin{aligned}
& -\frac{\partial f}{\partial t}+\frac{1}{\kappa^{2}} r^{2} \frac{\partial^{2} f}{\partial r^{2}}+r \frac{\partial f}{\partial r}=f\left(f^{2} r^{2}+S^{2}-r^{2}\right) \text { in }(\epsilon, R), \\
& \frac{\partial f}{\partial r}(\epsilon)=0 \text { and } \quad \frac{\partial f}{\partial r}(R)=0, \\
& r \frac{\partial^{2} S}{\partial r^{2}}-\frac{\partial S}{\partial r}=r f^{2} S \quad \text { in } \quad(\epsilon, R), \\
& S(\epsilon)=0 \text { and } \frac{1}{R} \frac{\partial S}{\partial r}(R)=H_{0},
\end{aligned}
$$

Vol. 16, n n $^{\circ}$ 6-1999. 
using the same scheme as in [1], that is implicit discretisation in time. It can be shown as in [6], thanks to the Maximum Principle, that a minimizer of the Ginzburg-Landau energy is an asymptotically stable solution of this problem. We compute the solutions with $R=1$. We study the convergence of $f_{\kappa}$ when $\kappa$ tends to infinity. Figure $1\left(H_{0}=1\right)$ and figure $2\left(H_{0}=3\right)$ illustrate the two different behaviours described in Theorem 2.7.

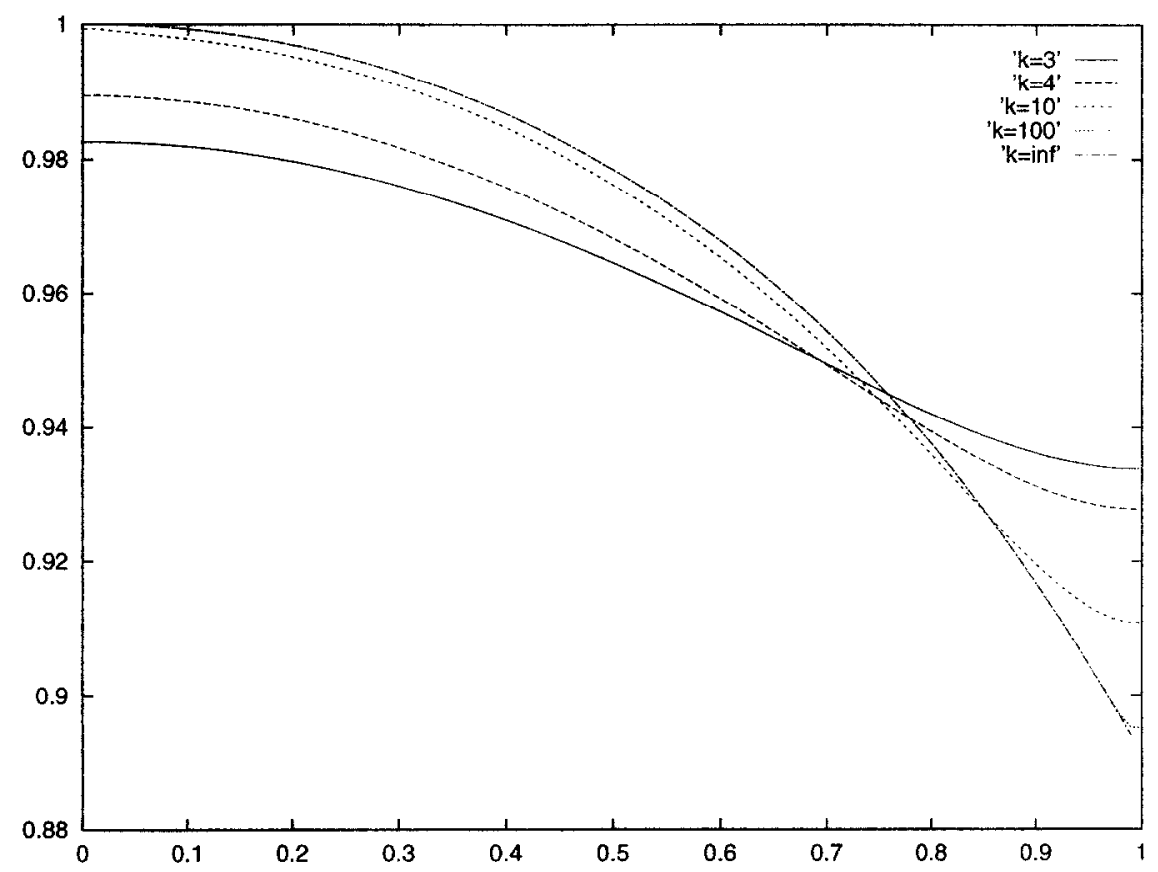

Figure 1. - Radial solutions $f(r)$ for $R=1, H_{0}=1$.

Remark. - It would be interesting to show that $f_{\kappa}$ is nonincreasing and improve the convergence of the sequence.

\section{THE CASE WITH VORTICES}

In this section, we allow $N$ vortices to appear in the center of the ball and intend to minimize the energy over this number $N$. We are going to show that $N \neq 0$ for the minimizer, and more precisely that $N / \kappa$ has a limit when $\kappa$ tends to infinity. The existence of vortices at the center of the ball can be described mathematically by introducing, as in [4], solutions 


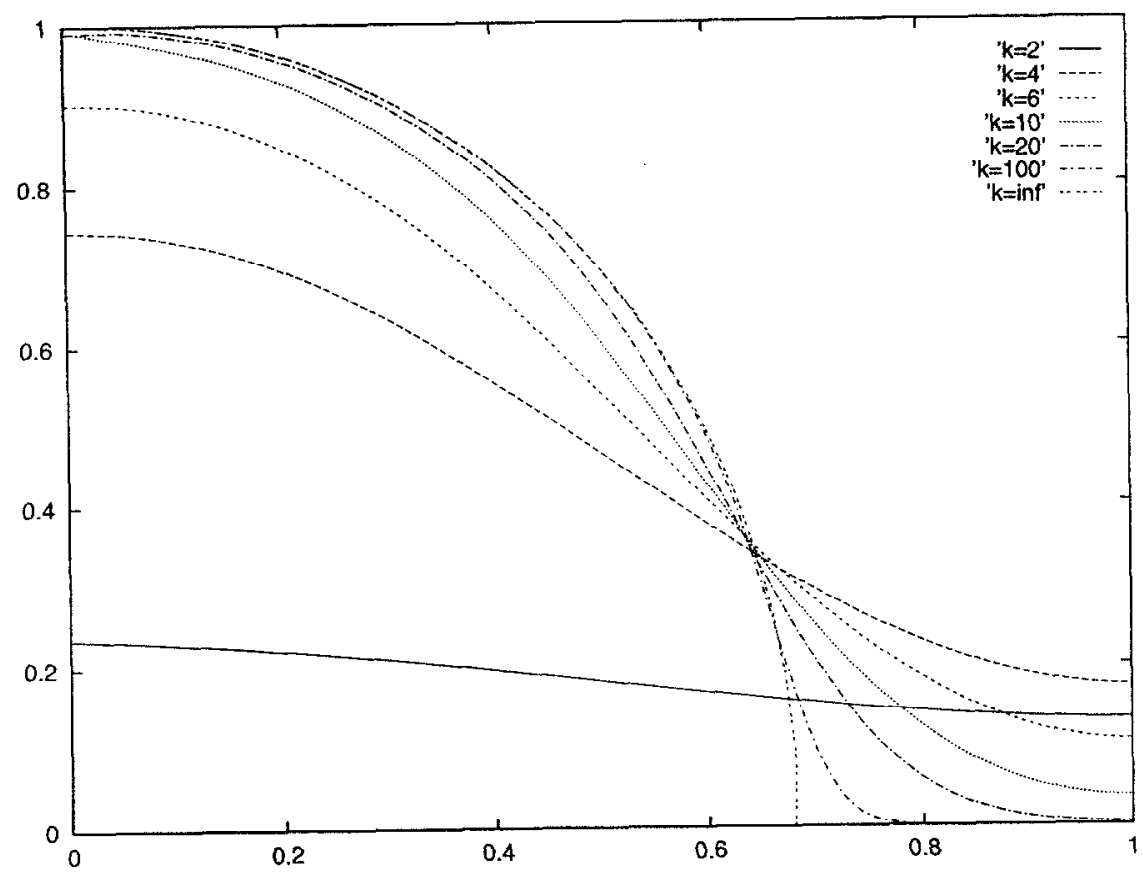

Figure 2. - Radial solutions $f(r)$ for $R=1, H_{0}=3$.

$(\psi, \mathbf{A})$ such that

$$
\psi(r, \theta)=f(r) e^{i N \theta} \quad f(r) \in \mathbb{R}, \quad \text { and } \quad \mathbf{A}(r, \theta)=A(r) \mathbf{e}_{\theta}
$$

and $A$ will be regular at the origin. We may notice that $(\psi, \mathbf{A})$ is gauge equivalent (see Definition 1.1$)$ to $(f, \mathbf{Q})$ with

$$
\mathbf{Q}(r)=\frac{1}{r}\left(S(r)-\frac{N}{\kappa}\right) \mathbf{e}_{\theta},
$$

where $S(r) / r$ is regular at the origin.

In this situation, the Ginzburg-Landau energy is the following:

$$
\begin{aligned}
E_{\kappa}(N, f, S) & =\int_{B_{R}}\left(\frac{1}{\kappa^{2}} f^{\prime 2}+\frac{1}{2}\left(f^{2}-1\right)^{2}\right. \\
& \left.+\frac{1}{r^{2}}\left(S-\frac{N}{\kappa}\right)^{2} f^{2}+\left(\frac{1}{r} S^{\prime}-H_{0}\right)^{2}\right) r d r d \theta
\end{aligned}
$$




\subsection{Finite Ginzburg-Landau Parameter}

The introduction of $N$ in the energy comes from a number of vortices, that is an integer. But in fact, the definition (22) has a meaning for any real number $N$. So for mathematical purposes, from now on, we will allow $N$ to vary in $\mathbb{R}$. We will see that the convergence properties and the limit would not be affected by restricting $N$ to lie in $\mathbb{Z}$, if a judiciously sequence of $\kappa$ is chosen.

\subsubsection{Fixed number of vortices}

To start with, we fix $N$ and want to minimize $E_{\kappa}$ on $D_{f} \times D_{S}$ where

$$
\begin{gathered}
D_{f}=\left\{f \text { radial, } f \geq 0 \text { a.e. } f \in H^{1}\left(B_{R}\right)\right\}, \\
D_{S}=\left\{S \text { radial, } \frac{1}{r} S \in L^{2}\left(B_{R}\right) \text { and } \frac{1}{r} S^{\prime} \in L^{2}\left(B_{R}\right)\right\} .
\end{gathered}
$$

With the norm $\left\|(1 / r) S^{\prime}\right\|_{L^{2}}, D_{S}$ is a Hilbert space (see [4]). We will not give the proof of the next three Theorems as it is almost the same as in the case treated in [4].

Theorem 3.1. - If $E_{\kappa}(N, f, S)<\infty$, then $f \in H^{1}(0, R),(1 / r) S^{\prime} \in$ $L^{2}(0, R)$ and $(1 / r)(S(r)-S(0)) \in L^{2}(0, R)$.

THEOREM 3.2. - We have the following regularity properties:

(i) $f \in D_{f} \Rightarrow f \in C^{0}(0, R)$,

(ii) $S \in D_{S} \Rightarrow S \in C^{0}(0, R)$ and $S(0)=0$,

(iii) $f \in D_{f}, S \in D_{S}$ and $E_{\kappa}(N, f, S)<\infty \Rightarrow f \in C^{0}[0, R]$ and $f(0)=0$.

THEOREM 3.3. - There exists a minimizer $(f, S)$ of $E_{\kappa}$. It is in $\left(C^{\infty}\left(B_{R}-\{0\}\right) \cap C^{2}\left(B_{R}\right)\right)^{2}$ and is a solution of:

$$
\begin{aligned}
& \frac{1}{\kappa^{2}} f^{\prime \prime}+\frac{1}{r \kappa^{2}} f^{\prime}=f\left(f^{2}+\frac{1}{r^{2}}\left(\frac{N}{\kappa}-S\right)^{2}-1\right) \text { in }(0, R), \\
& f^{\prime}(R)=0, \\
& S^{\prime \prime}-\frac{1}{r} S^{\prime}=\left(S-\frac{N}{\kappa}\right) f^{2} \text { in }(0, R), \\
& \frac{1}{R} S^{\prime}(R)=H_{0} .
\end{aligned}
$$


Remark. - With the expression of the laplacian in the radial case, equations (23) and (25) can be rewritten:

$$
\begin{aligned}
& \frac{1}{\kappa^{2}} \Delta f=f\left(f^{2}+\frac{1}{r^{2}}\left(S-\frac{N}{\kappa}\right)^{2}-1\right) \text { in } B_{R} \\
& \Delta S-\frac{2}{r^{2}}\left(x_{1} \partial_{1} S+x_{2} \partial_{2} S\right)=\left(S-\frac{N}{\kappa}\right) f^{2} \text { in } B_{R}
\end{aligned}
$$

THEOREM 3.4. - If $(f, S)$ is a minimizer of $E_{\kappa}$ such that $f$ is not identically zero, then $0<f \leq 1$ on $(0, R]$ and $S>0$ on $(0, R)$. Moreover, neither function is constant on a subinterval of $(0, R)$.

Proof. - Since the solutions are regular by Theorem 3.3, if they are constant on a subinterval, it means they are constant on $[0, R]$. But the boundary condition $S(0)=0$ and (23)-(25) show that $S$ cannot be constant and the only possibility for $f$ is 0 .

If $(f, S)$ is a minimizer of $E_{\kappa}$, so is $(|f|, S)$. Hence $(|f|, S)$ is a solution of the Ginzburg-Landau equations and $|f|$ is $C^{\infty}$ on $(0, R)$. So either $f \equiv 0$ or $f$ is never equal to zero.

Let us assume $\max _{r \in[0, R]} f(r)=f\left(r_{0}\right)>1$. As $f(0)=0, r_{0} \in(0, R]$. Equation (27) can be rewritten

$$
\frac{1}{\kappa^{2}} \Delta f-c(r) f=0
$$

on an interval around $r_{0}$, which does not contain 0 , and on which $f \geq 1$ so that $c(r)$ is positive. We choose the largest interval possible. Necessarily, either $f=1$ on the boudary or $r=R$ is the right end. The strong Maximum Principle implies that the maximum is reached on the boundary. But because of condition (26), it cannot be reached on $r=R$ and otherwise $f=1$ on the boundary, which is the minimum. So that $f$ remains smaller than 1 .

We know that $S(0)=0$. Let us assume that there exists $r_{0}$ in $(0, R]$ such that $\max _{r \in[0, R]} S(r)=S\left(r_{0}\right) \leq 0$. Equation (28) gives:

$$
\Delta S-\frac{2}{r^{2}}\left(x_{1} \partial_{1} S+x_{2} \partial_{2} S\right)-S f^{2} \leq 0
$$

We again apply the Maximum Principle on an interval around $r_{0}$. The minimum is reached on the boundary which means $r_{0}=R$. But condition (26) gives that $S^{\prime}(R)>0$ which contradicts the Hopf Lemma. So $S>0$ on $(0, R]$. 
THEOREM 3.5. - If $(f, S)$ is a minimizer of $E_{\kappa}$, then $S$ is non decreasing.

Proof. - We rewrite equation (28) as follows:

$\Delta\left(S-\frac{N}{\kappa}\right)-\frac{2}{r^{2}}\left(x_{1} \partial_{1}\left(S-\frac{N}{\kappa}\right)+x_{2} \partial_{2}\left(S-\frac{N}{\kappa}\right)\right)-\left(S-\frac{N}{\kappa}\right) f^{2}=0$.

The Maximum Principle implies that $S-(N / \kappa)$ cannot reach any positive maximum nor any negative minimum in the interior. Since $S(0)=0$, there are two possibilities:

- $S \leq(N / \kappa)$ on $(0, R)$.

So $S$ has no local minimum in $(0, R)$. As $S^{\prime}(R)>0$, it implies $S$ is non decreasing.

- $S(r)>(N / \kappa)$ for some $r$ in $(0, R)$.

Let $r_{0}$ be the first point where $S=\frac{N}{\kappa}$. Necessarily, $S^{\prime}\left(r_{0}\right)>0$ by the Hopf Lemma and as in the previous case, $S$ is non decreasing on $\left(0, r_{0}\right)$. Then the Maximum principle gives that $S-(N / \kappa)$ has no positive maximum in $\left(r_{0}, R\right)$. So $S$ is non decreasing on $\left(r_{0}, R\right)$.

Remark. - It would be interesting to study the monotonicity of $f$.

\subsubsection{Minimization of $E_{\kappa}$}

From now on, we intend to minimize $E_{\kappa}$ over $\mathbb{R} \times D_{f} \times D_{S}$, that is to find the best number of vortices to put at the origin. We will show that the presence of vortices luwer the energy.

THEOREM 3.6. - There exists a minimizer $(N, f, S)$ of $E_{\kappa} ;(f, S)$ is in $\left(C^{\infty}\left(B_{R} \backslash\{0\}\right) \cap C^{2}\left(B_{R}\right)\right)^{2}$ and is a solution of (23)-(24)-(25)-(26) with

$$
\int_{B_{R}} \frac{1}{r^{2}}\left(S-\frac{N}{\kappa}\right) f^{2}=0
$$

Proof. - Let $\left(n_{i}, f_{n_{i}}, S_{n_{i}}\right)$ be a minimizing sequence. For cach $n_{i}$, we can always replace $f_{n_{i}}$ and $S_{n_{i}}$ by a minimizer of $E_{\kappa}$ with fixed $N=n_{i}$ as in the previous section. Then $f_{n_{i}}$ is bounded in $H^{1}\left(B_{R}\right) \cap L^{\infty}\left(B_{R}\right)$ and $S_{n_{i}}$ is bounded in $D_{S}$. So, up to the extraction of a subsequence,

$$
\begin{aligned}
f_{n_{i}} & \rightarrow f \quad \text { weakly in } H^{1}, \text { a.e. and strongly in } L^{p} \text { for all finite } p, \\
\frac{1}{r} S_{n_{i}}^{\prime} & \rightarrow \frac{1}{r} S^{\prime} \quad \text { weakly in } L^{2}, \\
\frac{1}{r} S_{n_{i}} & \rightarrow \frac{1}{r} S \text { strongly in } L^{p},
\end{aligned}
$$


where $(f, S) \in D_{f} \times D_{S}$. Thanks to lower semi-continuity, we have

$$
\begin{aligned}
& \int_{B_{R}} \frac{1}{\kappa^{2}} f^{\prime 2}+\frac{1}{2}\left(f^{2}-1\right)^{2}+\left(\frac{1}{r} S^{\prime}-H_{0}\right)^{2} \\
& \quad \leq \liminf _{i \rightarrow \infty} \int_{B_{R}} \frac{1}{\kappa^{2}} f_{n_{i}}^{\prime 2}+\frac{1}{2}\left(f_{n_{i}}^{2}-1\right)^{2}+\left(\frac{1}{r} S_{n_{i}}^{\prime}-H_{0}\right)^{2} .
\end{aligned}
$$

If the limit $f$ is identically zero, equation (30) implies that the normal state $\left(f_{0}, S_{0}\right)$, with $f_{0} \equiv 0$ and $S_{0}^{\prime}(r)=H_{0} r$, is a minimizer of $E_{\kappa}$. Then the number $N$ of vortices does not intervene.

When $f$ is not identically zero, the sequence $n_{i}$ is bounded. Indeed, we have

$$
\left(\frac{n_{i}}{\kappa}\right)^{2} \int_{B_{R}} \frac{1}{r^{2}} f_{n_{i}}^{2} \leq 2 \int_{B_{R}} \frac{1}{r^{2}}\left(\frac{n_{i}}{\kappa}-S_{n_{i}}\right)^{2} f_{n_{i}}^{2}+2 \int_{B_{R}} \frac{1}{r^{2}} S_{n_{i}}^{2} f_{n_{i}}^{2},
$$

and since $S_{n_{i}}$ is in $D_{S}$, it implies $(1 / r) S \in L^{2}\left(B_{R}\right)$. As a consequence, we can assume $n_{i} \rightarrow N$, and $(N, f, S)$ is a minimizer of $E_{\kappa}$ because of (30) and

$$
\forall \alpha \quad \int_{B_{R} \backslash B_{\alpha}} \frac{1}{r^{2}}\left(S-\frac{N}{\kappa}\right)^{2} f^{2} \leq \liminf _{i \rightarrow \infty} \int_{B_{R} \backslash B_{\alpha}} \frac{1}{r^{2}}\left(S_{n_{i}}-\frac{n_{i}}{\kappa}\right)^{2} f_{n_{i}}^{2} .
$$

COROLlary 3.7. - When the normal state is not a minimizer, then the minimizing solution has vortices at the origin.

Proof. - When $f \neq \equiv 0$, equation (29) gives that $S-N / \kappa$ changes sign. The study in section 2 implies that $N$ is positive.

\subsection{Infinite Ginzburg-Landau Parameter}

We let formally $\kappa=\infty$ in the energy. The difference with the one dimensional case is that we shall have to find the constant $C$ (which comes from the term $N / \kappa$ ) which minimizes the energy. We define

$$
E_{\infty}(C, f, S)=\int_{B_{R}} \frac{1}{2}\left(f^{2}-1\right)^{2}+\frac{1}{r^{2}}(S-C)^{2} f^{2}+\left(\frac{1}{r} S^{\prime}-H_{0}\right)^{2} r d r d \theta
$$

THEOREM 3.8. - There exists a minimizer $\left(C_{\infty}, f_{\infty}, S_{\infty}\right)$ of $E_{\infty}$ over $\mathbb{R} \times L_{\text {rad }}^{4}\left(B_{R} ; \mathbb{R}^{+}\right) \times D_{S} . S_{\infty}$ is in $\left.C^{2}\left(B_{R}\right)\right)$ and $Q(r)=\left(S_{\infty}(r)-C_{\infty}\right) / r$ Vol. $16, n^{\circ}$ 6-1999. 
satisfies

$$
\begin{aligned}
& Q^{\prime \prime}+\frac{1}{r} Q^{\prime}-\frac{1}{r^{2}} Q=Q\left(1-Q^{2}\right) \mathbf{1}_{|Q| \leq 1}, \\
& Q^{\prime}(r)+\frac{1}{r} Q(r)=H_{0} \quad \text { when } \quad|Q(r)| \geq 1 \text { and } r=R \\
& \int_{B_{R}} Q\left(1-Q^{2}\right) \mathbf{1}_{|Q| \leq 1}=0 \\
& f_{\infty}^{2}=\left(1-Q^{2}\right) \mathbf{1}_{|Q| \leq 1} .
\end{aligned}
$$

Proof. - We call $\left(C_{n}, f_{n}, S_{n}\right)$ a minimizing sequence and define $Q_{n}(r)=\left(S_{n}(r)-C_{n}\right) / r$. We proceed as in the one dimensional case: replacing $f_{n}^{2}$ by $\left(1-Q_{n}^{2}\right) \mathbf{1}_{\left|Q_{n}\right| \leq 1}$ can only lower the energy so $Q_{n}$ is a minimizing sequence of

$$
J(Q)=\int_{B_{R}}\left(\frac{1}{2}-\frac{1}{2}\left(1-Q^{2}\right)^{2} \mathbf{1}_{|Q| \leq 1}\right)+\left(\frac{1}{r}(r Q)^{\prime}-H_{0}\right)^{2} r d r d \theta .
$$

Since $(1 / r)(r Q)^{\prime}$ is bounded in $L^{2},(1 / r) S_{n}^{\prime}$ is bounded in $L^{2}$ too, so it implies $S_{n}$ is bounded in $L^{\infty}$. As we are only concerned with $r$ such that $\left|Q_{n}(r)\right| \leq 1$, we can also assume $C_{n}$ is bounded. Then we can extract a subsequence that will converge to a minimizer of $E_{\infty}$. Equations (32) and (34) are the corresponding Euler-Lagrange equations for the variations of $S$ and $C$.

THEOREM 3.9. - Any minimizer $\left(C_{\infty}, f_{\infty}, S_{\infty}\right)$ of $E_{\infty}$ is such that $f_{\infty} \neq \equiv$. More precisely, let $Q_{\infty}(r)=\left(S_{\infty}(r)-C_{\infty}\right) / r$. Then $Q_{\infty}$ is increasing on $(0, R),\left|Q_{\infty}\right|$ remains smaller than one in an annulus $\left(r_{1}, R\right)$ with $Q_{\infty}\left(r_{1}\right)=-1$. On $\left(0, r_{1}\right), f_{\infty} \equiv 0$ and $Q_{\infty}(r)=(1 / 2) H_{0} r-(1 / r) C_{\infty}$, where $C_{\infty}=r_{1}\left(1+H_{0} r_{1} / 2\right)$.

Proof. - We are going to study the shape of solutions of (32)-(33)-(34)(35). An easy computation shows that if $Q^{\prime}(r)+(1 / r) Q(r)=H_{0}$, then $Q(r)=(1 / 2) H_{0} r-(1 / r) C$ for a given constant $C$. As (32) can be rewritten

$$
\Delta Q-Q\left(1+\frac{1}{r^{2}}-Q^{2}\right)=0 \text { when }|Q| \leq 1
$$

the Maximum Principle implies that $Q$ can neither reach a positive local maximum nor a negative local minimum while it remains between -1 and 1. Moreover (34) implies that $Q$ changes sign. So any solution of (32)(33)-(34)-(35) with $f \neq 0$ is such that $Q$ is increasing, $|Q|<1$ in an annulus 
and outside the annulus, the solution is defined by (33) and the continuity condition on the boundary. There are two possible types of solution:

- type $a:|Q|<1$ on $\left(r_{1}, R\right)$ with $Q\left(r_{1}\right)=-1$ and $Q(R)=\alpha \in(0,1)$,

- type $b:|Q|<1$ on $\left(r_{1}, r_{2}\right)$ with $Q\left(r_{1}\right)=-1, Q\left(r_{2}\right)=1$ and $r_{2}<R$.

We are going to show that type $b$ solutions cannot occur. Let us define

$$
F(r)=\frac{1}{2}\left(Q^{\prime}(r)+\frac{1}{r} Q(r)\right)^{2}+\frac{1}{4}\left(1-Q^{2}(r)\right)^{2},
$$

on the annulus where $|Q|<1$. Thanks to (32), we see that $F^{\prime}(r)=$ $(1 / r) Q^{2}\left(1-Q^{2}\right)$, so $F$ is increasing on the annulus. But this is impossible in the case of a type $b$ solution since $F\left(r_{1}\right)=F\left(r_{2}\right)=H_{0}^{2} / 2$. So we have to investigate the existence of type $a$ solutions. The proof now relies on a shooting method: for a given $\alpha \in(0,1)$, there is a unique solution $Q_{\alpha}$ of

$$
\left\{\begin{array}{l}
Q_{\alpha}^{\prime \prime}+\frac{1}{r} Q_{\alpha}^{\prime}-\frac{1}{r^{2}} Q_{\alpha}=Q_{\alpha}\left(1-Q_{\alpha}^{2}\right) \quad \text { on } \quad(0, R), \\
Q_{\alpha}(R)=\alpha \quad \text { and } \quad Q_{\alpha}^{\prime}(R)=H_{0}-\frac{\alpha}{R}
\end{array}\right.
$$

We check that (33) is satisfied when $r=R$. We are interested in the interval where $Q_{\alpha}$ remains smaller than 1 . We introduce the same sets as in the proof of Theorem 2.5: $I\left(R, H_{0}\right), I_{0}\left(R, H_{0}\right)$ and $I_{1}\left(R, H_{0}\right)$. Notice that the Maximum Principle applied to (37) implies that when $\alpha \in I_{0}\left(R, H_{0}\right)$ there exists $r_{1, \alpha} \in(0, R)$ with $Q_{\alpha}\left(r_{1, \alpha}\right)=-1$ and $Q_{\alpha}$ is increasing on $\left(r_{1, \alpha}, R\right)$. For $\alpha \in I_{0}\left(R, H_{0}\right)$, we define

$$
f_{R}(\alpha)=H_{0}+\frac{1}{r_{1, \alpha}}-Q_{\alpha}^{\prime}\left(r_{1, \alpha}\right)
$$

We notice thanks to (32) that

$$
f_{R}(\alpha)=\int_{B_{R}} Q_{\alpha}\left(1-Q_{\alpha}^{2}\right) \mathbf{1}_{\left|Q_{\alpha}\right| \leq 1}=\int_{r_{1, \alpha}}^{R} r Q_{\alpha}\left(1-Q_{\alpha}^{2}\right) d r
$$

Classical ODE theory implies that $f_{R}$ is a continuous function of $\alpha$.

lst step. - Let $R$ and $H_{0}$ bc fixed. Wc are going to show that when $I\left(R, H_{0}\right) \neq \emptyset$, there is a minimizer of $E_{\infty}$ of type $a$. In this case, we know from the proof of Theorem 2.5 that $I_{0}\left(R, H_{0}\right)=\left(0, \alpha^{*}\right)$ with $\alpha^{*} \in I\left(R, H_{0}\right)$. For $\alpha=0, Q_{\alpha}<0$ on $\left(r_{1, \alpha}, R\right)$ so (40) gives that $f_{R}(0)<0$. As a consequence, $f_{R}$ is negative for $\alpha$ small. 
We let $\alpha$ tend to $\alpha^{*}$. We call $r_{0, \alpha}$ the point where $Q_{\alpha}(r)=0$. Up to the extraction of a subsequence, we have $r_{0, \alpha} \rightarrow r_{0}$. If $r_{0}>0$, then classical ODE estimates give that $Q_{\alpha}\left(r_{0, \alpha}\right) \rightarrow Q_{\alpha^{*}}\left(r_{0}\right)$. But as $\alpha^{*} \in I\left(R, H_{0}\right)$, we cannot have $Q_{\alpha^{*}}\left(r_{0}\right)=0$ for $r_{0}>0$, so $r_{0}=0$. Since $Q_{\alpha}$ is increasing, $r_{1, \alpha}<r_{0, \alpha}$ and $r_{1, \alpha} \rightarrow 0$ too. We know that $Q_{\alpha}$ is bounded on $\left(r_{1, \alpha}, R\right)$, so we have

$\lim _{\alpha \rightarrow \alpha^{*}} \int_{r_{1, \alpha}}^{r_{0, \alpha}} r Q_{\alpha}\left(1-Q_{\alpha}^{2}\right) d r=0 \quad$ and $\quad \lim _{\alpha \rightarrow \alpha^{*}} \int_{r_{0, \alpha}}^{R} r Q_{\alpha}\left(1-Q_{\alpha}^{2}\right) d r>0$.

It implies that $f_{R}(\alpha)>0$ for $\alpha$ close to $\alpha^{*}$. As $f_{R}(\alpha)<0$ for small $\alpha$, there exists $\beta \in\left(0, \alpha^{*}\right)$ such that $f(\beta)=0$. So $Q_{\beta}$ is a solution to (32)-(33)-(34).

We already know that the minimizer of $E_{\infty}$ exists. Either it is the normal state, that is a solution $\left(C_{0}, f_{0}, S_{0}\right)$ with $f_{0} \equiv 0$ and $S_{0}^{\prime}=H_{0} r$, or a solution of type $a$. We may notice that once we have a solution $Q$ of type $a$, we can go back to $(C, f, S)$ thanks to $(35)$, the continuity condition $H_{0} r_{1} / 2-C / r_{1}=-1$ and the definition of $Q$ which gives $S(r)=C+r Q(r)$. Moreover, $E_{\infty}(C, f, S)=J\left(Q_{\beta}\right)$. Now let us show that $Q_{\beta}$ provides a minimizer of $E_{\mathrm{w}}$. We introduce a new energy

$$
E(r)=\frac{1}{2}\left(r Q_{\beta}^{\prime}\right)^{2}-\frac{1}{2} Q_{\beta}^{2}+\frac{1}{4} r^{2}\left(1-Q_{\beta}^{2}\right)^{2} .
$$

Computing $E^{\prime}(r)$, using equation (32), gives

$$
\frac{1}{2} \int_{r_{1, \beta}}^{R} r\left(1-Q_{\beta}^{2}\right)^{2} d r=\frac{1}{4} R^{2}\left(1-\beta^{2}\right)^{2}+\frac{1}{2} H_{0}^{2}\left(R^{2}-r_{1, \beta}^{2}\right)-H_{0}\left(R \beta+r_{1, \beta}\right) .
$$

This and an integration by parts on $\int_{r_{1, \beta}}^{R}(1 / r)\left(\left(r Q_{\beta}\right)^{\prime}\right)^{2}$ enable us to estimate $J\left(Q_{\beta}\right)$.

$$
J\left(Q_{\beta}\right)=\frac{1}{4} R^{2}-\frac{1}{4} R^{2}\left(1-\beta^{2}\right)^{2}-\int_{r_{1, \beta}}^{R} r Q_{\beta}^{2}\left(1-Q_{\beta}^{2}\right) .
$$

Since $E_{\infty}\left(C_{0}, f_{0}, S_{0}\right)=R^{2} / 4$, it means $Q_{\beta}$ has a lower energy than the normal state.

2nd step. - We assume $H_{0}$ is fixed. Then for small $R\left(R \leq 1 / H_{0}\right.$ for instance), the first step indicates that there exist a minimizer of type $a$. We call

$$
R_{0}=\max \left\{R \text { st the normal state is not a minimizer of } E_{\infty} \cdot\right\}
$$


Let us assume that $R_{0}$ is finite. A straightforward argument shows that if $Q_{\alpha_{R}}$ corresponds to a minimizer of $E_{\infty}^{\top}$ with $Q_{\alpha_{R}}(R)=\alpha_{R}$, and if $J_{0}=R_{0}^{2} / 4$ is the energy of the normal state for $R=R_{0}$, then

$$
\lim _{R \rightarrow R_{0}} J\left(Q_{\alpha_{R}}\right)=J_{0}
$$

According to (41), it means that $\alpha_{R} \rightarrow 1$ and $\int_{r_{1, \alpha_{R}}}^{R} r Q_{\alpha_{R}}^{2}\left(1-Q_{\alpha_{R}}^{2}\right) \rightarrow 0$. But this is impossible, so $R_{0}=+\infty$.

Remark. - Numerical computations show that $f_{R}$ is increasing on $(0,1)$ hence the minimizer of $E_{\infty}$ is unique.

COROLlaRY 3.10. - For all $H_{0}$, there exists $\kappa_{0}$ such that for $\kappa \geq \kappa_{0}$, the normal state is not a minimizer of $E_{\kappa}$.

The proof relies on energy comparisons as in [1].

Proposition 3.11. - When $R$ is large, $C_{\infty}$ is equivalent to $H_{0} R^{2} / 2$.

Proof. - We already know that $C_{\infty}=r_{1}\left(1+H_{0} r_{1} / 2\right)$, where $r_{1}$ is such that $Q\left(r_{1}\right)=-1$ and $Q$ is as in Theorem 3.8 associated to the minimizer of $E_{\infty}$. We only need to show that $r_{1}$ is equivalent to $R$ when $R$ is large. Let $V$ be the solution of

$$
\left\{\begin{array}{l}
V^{\prime \prime}=V\left(1-V^{2}\right) \quad \text { on } \quad(0, R) \\
V(R)=\alpha \quad \text { and } \quad V^{\prime}(R)=H_{0}-\alpha / R
\end{array}\right.
$$

where $\alpha=Q(R)$. It is easy to see that there exists $\rho_{1}$ in $(0, R)$ such that $V\left(\rho_{1}\right)=-1, V$ is increasing on $\left(\rho_{1}, R\right)$ and the energy $V^{\prime 2}+\left(1-V^{2}\right)^{2} / 2$ is preserved on $\left(\rho_{1}, R\right)$. A straightforward computation gives

$$
R-\rho_{1}=\int_{-1}^{\alpha}\left(\left(H_{0}-\frac{\alpha}{R}\right)^{2}+\frac{1}{2}\left(1-\alpha^{2}\right)^{2}-\frac{1}{2}\left(1-v^{2}\right)^{2}\right)^{-\frac{1}{2}} d v .
$$

So if we show that $r_{1}>\rho_{1}$, the proof is over. We know that $Q<V$ for $r$ close to $R$ and we are going to show that $Q$ and $V$ cannot intersect before reaching -1 . Let

$$
E_{1}(r)=Q^{\prime 2}+\frac{1}{2}\left(1-Q^{2}\right)^{2}
$$

We immediately get $E_{1}^{\prime}(r)=\left(2 / r^{2}\right) Q^{\prime}\left(Q-r Q^{\prime}\right)$. Let $r_{0}$ be the point where $Q$ crosses zero. Since $Q$ is increasing, $E_{1}^{\prime}<0$ on $\left(r_{1}, r_{0}\right)$. Let $E_{2}(r)=r Q-r 2 Q^{\prime}$ on $\left(r_{0}, R\right)$. Since $E_{2}^{\prime}(r)=-r^{2} Q\left(1-Q^{2}\right)$, it implies 
$E_{2}(r)<0$ on $\left(r_{0}, R\right)$ and $E_{1}$ is decreasing. Since for $r=R$ the energy $E_{1}$ is the same for $Q$ and $V$, it means

$$
{Q^{\prime}}^{2}+\frac{1}{2}\left(1-Q^{2}\right)^{2}>V^{\prime 2}+\frac{1}{2}\left(1-V^{2}\right)^{2} \quad \forall r \in\left(r_{1}, R\right) .
$$

So $Q$ and $V$ cannot intersect before reaching -1 and $r_{1}>\rho_{1}$.

\subsection{Convergence of minimizers}

THEOREM 3.12. - Let $\left(N_{\kappa}, f_{\kappa}, S_{\kappa}\right)$ be a sequence of minimizers of $E_{\kappa}$. There exists $\left(C_{\infty}, f_{\infty}, S_{\infty}\right)$, a minimizer of $E_{\infty}$, and a subsequence $\left(N_{\kappa_{n}}, f_{\kappa_{n}}, S_{\kappa_{n}}\right)$, such that when $\kappa_{n}$ tends to $\infty$

$$
\begin{aligned}
& \frac{N_{\kappa_{n}}}{\kappa_{n}} \rightarrow C_{\infty}, \\
& f_{\kappa_{n}} \rightarrow f_{\infty} \text { in } L^{p}\left(B_{R}\right) \text { for all finite } p, \\
& \frac{1}{r} S_{\kappa_{n}} \rightarrow \frac{1}{r} S_{\infty} \text { in } C^{0, \alpha}\left(B_{R}\right) \text { for all } \alpha \in(0,1) .
\end{aligned}
$$

Proof. - Let $\left(C_{\infty}, f_{\infty}, S_{\infty}\right)$ be a minimizer of $E_{\infty}$. We have seen that $f_{\infty}$ is not in $H^{1}\left(B_{R}\right)$, but as in [1], we can find $g_{\kappa}$ in $H^{1}\left(B_{R}\right)$ such that

$$
\lim _{\kappa \rightarrow \infty} E_{\kappa}\left(C_{\infty} \kappa, g_{\kappa}, S_{\infty}\right)=E_{\infty}\left(C_{\infty}, f_{\infty}, S_{\infty}\right)
$$

Let $\left(N_{\kappa}, f_{\kappa}, S_{\kappa}\right)$ be a minimizer of $E_{\kappa}$. Energy comparisons give:

$$
\begin{gathered}
E_{\infty}\left(C_{\infty}, f_{\infty}, S_{\infty}\right) \leq E_{\infty}\left(\frac{N_{\kappa}}{\kappa}, f_{\kappa}, S_{\kappa}\right) \leq E_{\kappa}\left(N_{\kappa}, f_{\kappa}, S_{\kappa}\right) \\
E_{\kappa}\left(N_{\kappa}, f_{\kappa}, S_{\kappa}\right) \leq E_{\kappa}\left(C_{\infty} \kappa, g_{\kappa}, S_{\infty}\right) .
\end{gathered}
$$

We let $\kappa$ tend to infinity and obtain

$$
\begin{gathered}
\lim _{\kappa \rightarrow \infty} \int_{B_{R}} \frac{1}{\kappa^{2}}{f^{\prime}}_{\kappa}^{2}=0 \\
\text { and } \lim _{\kappa \rightarrow \infty} E_{\infty}\left(\frac{N_{\kappa}}{\kappa}, f_{\kappa}, S_{\kappa}\right)=E_{\infty}\left(C_{\infty}, f_{\infty}, Q_{\infty}\right) .
\end{gathered}
$$

It implies that $(1 / r) S_{\kappa}$ is bounded in $D_{S}$. As $\left\|f_{\kappa}\right\|_{L^{\infty}} \leq 1$, up to the extraction of a subsequence,

$$
\begin{aligned}
& f_{\kappa}^{2} \rightarrow f^{2} \quad \text { weakly in } L^{2}\left(B_{R}\right), \\
& \frac{1}{r} S_{\kappa}^{\prime} \rightarrow \frac{1}{r} S^{\prime} \quad \text { weakly in } L^{2}, \\
& \frac{1}{r} S_{\kappa} \rightarrow \frac{1}{r} S \quad \text { strongly in } L^{p} \text { for all finite } p
\end{aligned}
$$


where $(f, S) \in L^{4}\left(B_{R}\right) \times D_{S}$. Weak convergence and lower semi continuity give

$$
\int_{B_{R}} \frac{1}{2}\left(f^{2}-1\right)^{2}+\left(\frac{1}{r} S^{\prime}-H_{0}\right)^{2} \leq \liminf _{\kappa \rightarrow \infty} \int_{B_{R}} \frac{1}{2}\left(f_{\kappa}^{2}-1\right)^{2}+\left(\frac{1}{r} S_{\kappa}^{\prime}-H_{0}\right)^{2} .
$$

(i) If $f \equiv 0$, it is easy to see that $f_{\kappa} \rightarrow 0$ a.e. and in $L^{p}$ for all finite $p$. Then (44) and (45) imply

$$
E_{\infty}(0, f, S) \leq \liminf _{\kappa \rightarrow \infty} E_{\kappa}\left(N_{\kappa}, f_{\kappa}, S_{\kappa}\right)=E_{\infty}\left(C_{\infty}, f_{\infty}, S_{\infty}\right)
$$

So it means that the normal state is a minimizer of $E_{\infty}$, which is not the case as shown in Theorem 3.9.

(ii) So $f \neq 0$, and as in the proof of Theorem 3.6, since

$$
\left(\frac{N_{\kappa}}{\kappa}\right)^{2} \int_{B_{R}} \frac{1}{r^{2}} f_{\kappa}^{2}
$$

is bounded independently of $\kappa$, then the scquence $N_{\kappa} / \kappa$ is bounded. So, up to the extraction of a subsequence, $N_{\kappa} / \kappa \rightarrow C$. Let $\mathbf{A}_{\kappa}(r)=(1 / r) S_{\kappa}(r) \mathbf{e}_{\theta}$. We have

$$
\Delta \mathbf{A}_{\kappa}=\left(\mathbf{A}_{\kappa}-\frac{N_{\kappa}}{\kappa r} \mathbf{e}_{\theta}\right) f_{\kappa}^{2}
$$

Since $\mathbf{A}_{\kappa}$ is bounded in $H^{1}$ and $\Delta \mathbf{A}_{\kappa}$ is bounded in $L^{p}$ for $p<2$, it implies that $\mathbf{A}_{\kappa}$ is bounded in $W^{2, p}$ and we infer from elliptic estimates that $\mathbf{A}_{\kappa}$ converges to $\mathbf{A}$ in $W^{1, p}\left(B_{R}\right)$ for $p<2$ and $C^{0, \alpha}\left(B_{R}\right)$ for $\alpha \in(0,1)$. For all small $\epsilon$, we have

$$
\liminf _{\kappa \rightarrow \infty} \int_{B_{R} \backslash B_{\epsilon}} \frac{1}{r^{2}}\left(S_{\kappa}-\frac{N_{\kappa}}{\kappa}\right)^{2} f_{\kappa}^{2} \geq \int_{B_{R} \backslash B_{\epsilon}} \frac{1}{r^{2}}(S-C)^{2} f^{2} .
$$

We can easily derive from (44)-(45)-(46) that $(C, f, S)$ is a minimizer of $E_{\infty}$. So thanks to Theorem 3.9 , there exists $r_{1}$ such that $f \equiv 0$ on $\left(0, r_{1}\right)$ and $f^{2}=1-(S-C)^{2} / r^{2}$ on $\left(r_{1}, R\right)$. Then we proceed as in [1] to show that $f_{\kappa}$ tends to $f$ strongly in $L^{p}$, now that we know the shape of $f$.

\subsection{Numerical Study}

We use the same scheme as before. We compute the solutions with $R=1$ and $H_{0}=3$. We are interested in what happens with large $\kappa$. Figure 3 


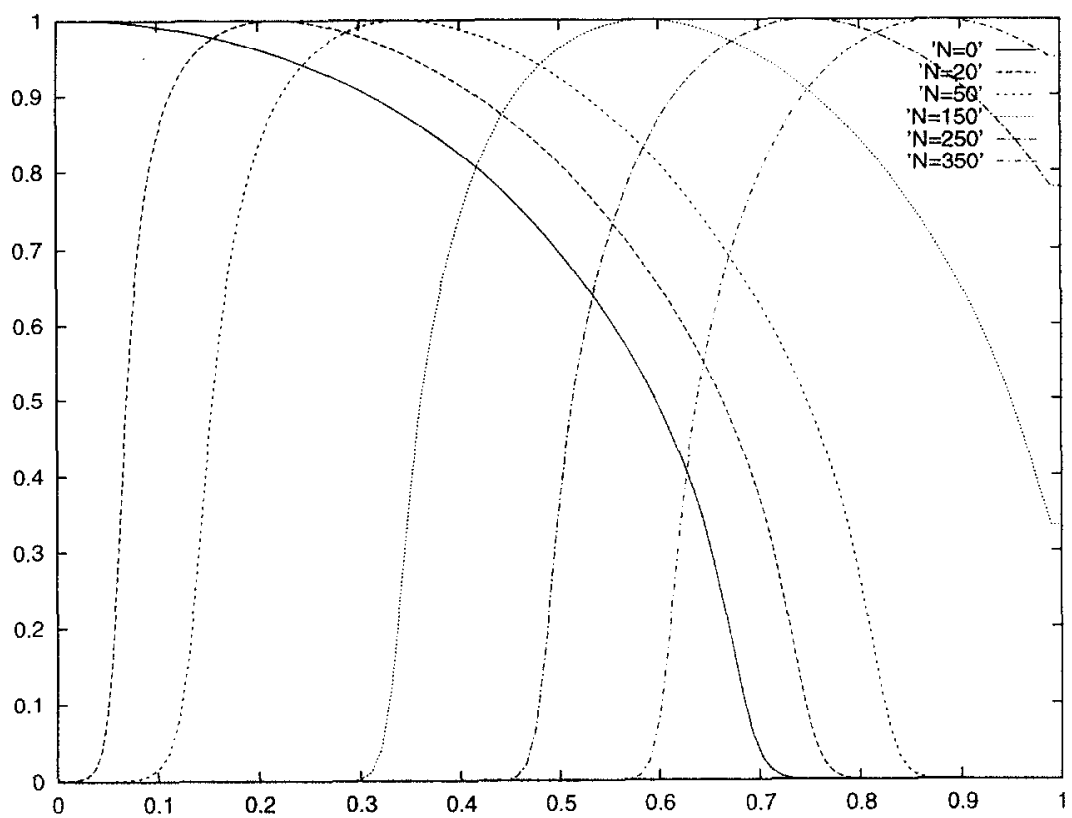

Figure 3. - Radial solutions with vortices $f(r)$ for $R=1, k=300, H_{0}=3$.

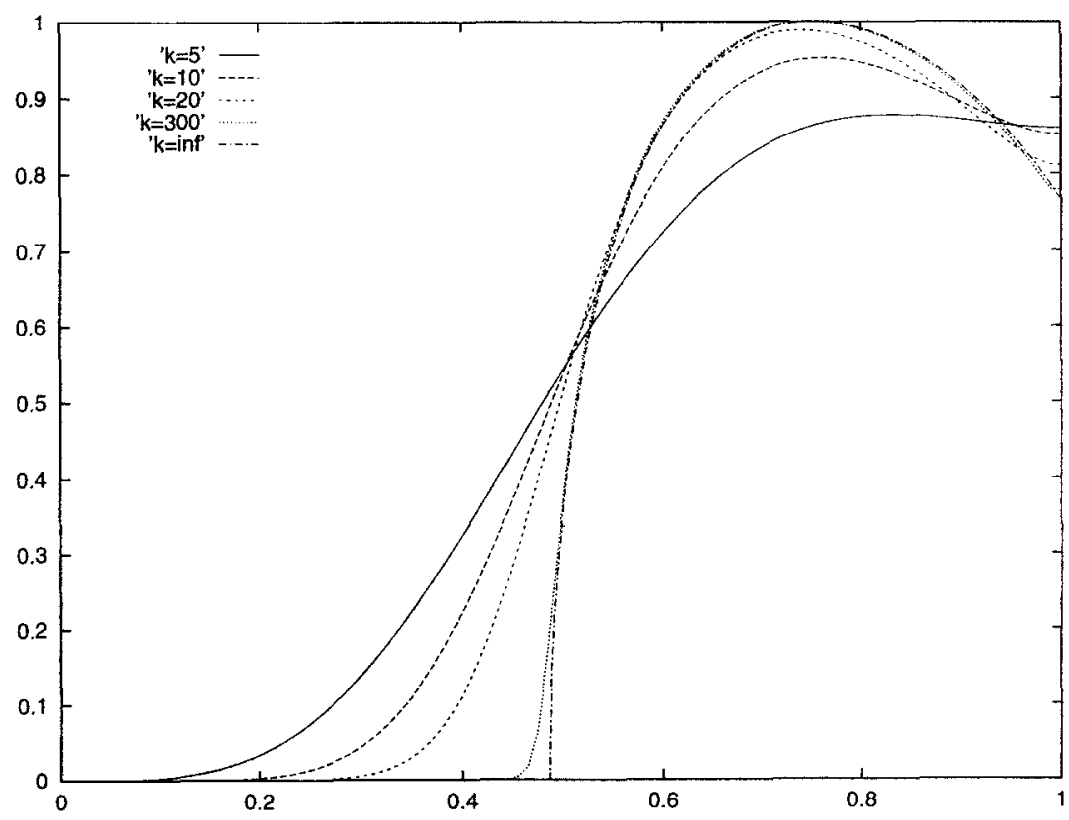

Figure 4. - Minimizers of $E_{\kappa}$ with vortices $f(r)$ for $R=1, H_{0}=3$. 
illustrates the different types of radial solutions $f$ to the Ginzburg-Landau equations according to the number of vortices:

- when $N$ is too large, the only solution is $f \equiv 0$;

- for $N$ of the same order as $\kappa, f$ is equal to zero in a small ball around the origin;

- for small $N, f$ is equal to zero in an exterior ring.

Figure 4 illustrates Theorem 3.12: it shows the convergence of minimizers $f_{\kappa}$ as $\kappa$ tends to infinity. We can see that the vortex core in an inside ball and there is an outside annulus where superconductivity remains.

\section{CONCLUSION}

We have proved in the case of a ball, that for large $\kappa$, the minimizer among radially symmetric solutions has $N$ vortices concentrated at the origin, $N$ being of order $\kappa$. It should be interesting to make a stability analysis of these minimizers. What we may expect for the global minimizer of $E_{\kappa}$ is to have of order $\kappa$ vortices, but not necessarily concentrated at one point.

\section{ACKNOWLEDGMENTS}

The author is very grateful to $\mathrm{H}$. Berestycki and S. J. Chapman for helpful discussions.

\section{REFERENCES}

[1] A. Aftalion, On the minimizers of the Ginzburg-Landau energy for high kappa: the one-dimensional case, EJAM, Vol. 8, 1997, pp. 331-345.

[2] P. Baumann, D. Phillips and Q. TANG, Stable nucleation for the Ginzburg-Landau system with an applied magnetic field, Preprint, 1996.

[3] H. Berestycki, A. Bonnet and S. J. Chapman, A semi-elliptic system arising in the theory of superconductivity, Comm. Appl. Nonlinear Anal., Vol. 1, 3, 1994, pp. 1-21.

[4] M. S. Berger and Y. Y. CHEN, Symmetric Vortices for the Ginzberg-Landau Equations of Superconductivity and the Nonlinear Desingularisation Phenomenon, J. Func. Anal., Vol. 82, 1989, pp. 259-295.

[5] F. Bethuel, H. Brezis and F. Helein, Ginzburg-Landau Vortices, Birkhäuser, 1994.

[6] C. Bolley, Solutions numériques de problèmes de bifurcation, RAIRO Anal. Num., Vol. 14, 1980 , pp. $127-147$.

[7] S. J. Chapman; Nucleation of superconductivity in decreasing fields I, Europ. J. Appl. Math., Vol. 5, 1994, pp. 449-468.

[8] S. J. Chapman, Nucleation of superconductivity in decreasing fields II, Europ. J. Appl. Math., Vol. 5, 1994, pp. 469-494. 
[9] S. J. CHAPMAN, A mean-field model of superconducting vortices in three dimensions, SIAM J. Appl. Math., Vol. 55, 1995, pp. 1259-1274.

[10] S. J. Chapman, Motion of vortices in type II superconductors, SIAM J. Appl. Math., Vol. 55, 1995, pp. 1275-1296.

[11] S. J. Chapman, S. D. Howison and J. R. Ockendon, Macroscopic models of superconductivity, SIAM Review, Vol. 34, 4, 1992, pp. 529-560.

[12] Y. Y. CHEN, Nonsymmetric vortices for the Ginzberg-Landau equations on the bounded domain, J. Math. Phys., Vol. 30, 1989, pp. 1942-1950.

[13] Q. Du, M. D. Gunzburger and J. S. PETERSON, Analysis and approximation of the Ginzburg-Landau model of superconductivity, SIAM Review, Vol. 34, 1, 1992, pp. 54-81.

[14] C. M. Elliot, H. Matano and Tang QI, Zeros of a complex Ginzburg-Landau order parameter with applications to superconductivity, Furop. J. Appl. Math., Vol. 5, 1994, pp. 431-448.

[15] D. Gilbarg and N. S. Trudinger, Elliptic Partial Differential Equations of Second Order, 2nd edition, Springer, Berlin, 1983.

[16] V. L. GinzBuRG and L. D. LANDau, On the theory of superconductivity, Soviet Phys. JETP, Vol. 20, 1950, p. 1064

[17] H. G. KAPER and M. K. KWONG, Uniqueness of non-negative solutions of semilinear elliptic equations, Nonlinear Diffusion Equations and Their Equilibrium States, II, W. M. Ni, L. A. Peletier and J. Serrin (eds.), MSRI Conf. Proc., Springer-Verlag, New York, 1988, pp. $1-17$.

(Manuscript received April 17, 1996;

Revised version July 17, 1997.) 